\title{
David A. Clark: Cognitive-Behavioral Therapy for OCD and Its Subtypes: Second Edition
}

\author{
The Guilford Press, New York, 2020, 436 pages, 21 tables, 26 forms, 11 figures, Retails for \$35 \\ from Guilford Press
}

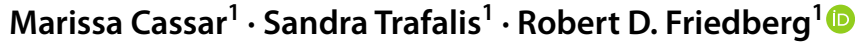

Accepted: 7 September 2020 / Published online: 11 September 2020

(c) Springer Science+Business Media, LLC, part of Springer Nature 2020

With jam-packed pages filled with valuable theoretical foundations, empirical findings, and nuggets of clinical wisdom, David A. Clark's guide on obsessive-compulsive disorders (OCD) presents the latest research and psychotherapeutic practices. This manual is a treasure chest of all things OCD, seamlessly flowing from topics regarding the nature of the disorder, current research and diagnostic classification, and insight into Cognitive-Behavioral Therapy (CBT) treatment for a range of OCD subtypes. Clark provides thorough literature reviews and does all of the work for the reader, making this handbook a one-stop-shop for clinical and research inquiries. The guide combines insights regarding case formulation, assessment, treatment, and portable tips for practitioners. Many tables and figures illustrate the phenomenology of the disorder and handy user-ready forms are sprinkled throughout the book. These forms can later be conveniently downloaded through access to a Web page, an updated feature to this second edition. For those who need quick-hand information, each chapter ends with concise bullet-pointed summaries for easy reference, and a full index may also aid in "think on your toes" situations.

Clark, widely known as a pioneer and leading expert in OCD, shares his acumen and clinical know-how in an accessible manner. While many similar manuals are monotonous reads and burdened by dense text material, Clark provides thought-provoking case examples through clear sections with multiple rich and relatable patient examples. These cases amplify the foundational science that buttress the

Robert D. Friedberg

rfriedberg@paloaltou.edu

1 Center for the Study and Treatment of Anxious Youth at Palo Alto University, Palo Alto, CA, USA interventions. Rather than being a dull slog through muddied wording, reading this book was like a brisk walk in the park!

This manual explores many specific topics regarding OCD, with attention to nearly every imaginable possible clinical feature. The work begins with foundations for understanding OCD and thoroughly explains diagnostic features, and etiology of the disorder. The text gets down to the nitty gritty, with useful definitions and examples of compulsions, obsessions, neutralization, and the role of control in OCD-related disorders. Specific to this second edition, Clark reviews 15 years of recent advances in clinical and research findings with heightened detail and utility of information. These updates are especially pivotal when reviewing exposure and response prevention techniques as well as updates to the cognitive-behavioral model. The text swiftly progresses into the fundamentals of CBT for OCD, with abridged mini-manuals surrounding topics of therapeutic rapport, assessment, case conceptualization, psychoeducation, multiple interventions, and empirical hypothesis-testing experiments. These sections are brimming with vital extensive role-play vignettes and step-by-step methods expanded from the first edition. The descriptions are so clear you could almost see it happening in your mind's eye.

The manual ends with uniquely fresh and innovative insights into subtype protocols covered with lavish attention to clinical utility. Clark begins with a comprehensive section on contamination, then moves into doubt and checking subtypes. These sections alone may be most valuable to those beginning to understand and work with OCD disorders, as Clark consolidates extensive research and knowledge into readable pages. The subtype section then moves into harm, sex, and religious obsessions, and finally leads into subtypes of symmetry, ordering, and arranging. These chapters explicitly educate the reader on some of these uncharted territories. 
In short, Clark has provided a masterful guide that beginning and experienced clinicians as well as graduate students should heed for advice. A compact, user-friendly, and advice-laden text, this manual is a must have for anyone planning to work with OCD related disorders. This guide is appropriate for many professionals in various disciplines including clinical psychology, counseling, psychiatry and social work. Additionally, the text is suitable as primary or supplementary text for courses related to evidence-based therapy, OCD and anxiety disorders, and possibly psychopathology and psychodiagnosis. In the current state of this world via the impact of COVID-19, hypochondriasis and
OCD-related rituals may be seemingly on a rise, and this guide may be essential for clinicians working in a postpandemic era. In conclusion, this guide is a phenomenal resource that should have a permanent home on the bookshelves of all clinicians working with these disorders.

Publisher's Note Springer Nature remains neutral with regard to jurisdictional claims in published maps and institutional affiliations. 